\title{
IMPACT OF INFANT PNEUMOCOCCAL VACCINATION ON INVASIVE PNEUMOCOCCAL DISEASES IN FRANCE, 2001-2006
}

\author{
A Lepoutre (a.lepoutre@invs.sante.fr) ${ }^{1}$, E Varon ${ }^{2}$, S Georges ${ }^{1}$, L Gutmann², Daniel Lévy-Bruhl ${ }^{1}$ \\ 1. Department of infectious diseases, Institut de Veille Sanitaire, Saint Maurice, France \\ 2. National Reference Centre for Pneumococci (NRCP), Georges Pompidou European Hospital, Paris, France
}

Vaccination with the 7-valent pneumococcal conjugate vaccine (PCV) has been recommended in France since 2003 for children under the age of two years who are at risk due to medical or living conditions. From 2006, the recommendation has been extended to all children under two years. The impact of PCV introduction on the incidence of pneumococcal meningitis and bacteraemia and on the serotype distribution in French children and other age-groups was assessed using laboratory surveillance data. The coverage with three doses of PCV was $44 \%$ in children aged 6-12 months in 2006. From $2001 / 2002$ to 2006 , the incidence of pneumococcal meningitis decreased from 8.0 to 6.0 cases per 100,000 , and the incidence of pneumococcal bacteraemia decreased from 21.8 to 17.5 cases per 100,000 in children under the age of two years. For the vaccine strains, the incidence of pneumococcal meningitis and bacteraemia decreased from 20,4 to 6.0 cases per 100,000 , while the incidence of pneumococcal meningitis and bacteraemia due to non-vaccine strains increased from 9.4 to 17.5 cases per 100,000 in this time period. The incidence in older children and adults did not decrease.

Further expansion of PCV coverage is expected to increase the impact of the vaccination in both children and adults. However, the fact that cases caused by vaccine serotypes have been partially substituted by cases of non-vaccine serotypes is likely to reduce the overall benefit of PCV in France, should this early observation be confirmed in the future.

\section{Introduction}

Streptococcus pneumoniae causes a wide spectrum of diseases, ranging from upper respiratory tract infections to severe invasive diseases. S. pneumoniae is the main cause of bacterial meningitis in France $[1,2]$. Invasive pneumococcal diseases (IPD) are more frequent in young children and the elderly and are associated with high case fatality ratio. The fatality ratio for pneumococcal meningitis has been estimated at $11 \%$ in children under the age of two years in a recent French study [3].

Two pneumococcal vaccines are currently licensed in Europe. The 23-valent pneumococcal polysaccharide vaccine was licensed in the 1980s and, although recommended for high risk individuals and elderly in many European countries, is poorly immunogenic in children under two years of age [4]. The 7-valent pneumococcal conjugate vaccine (PCV) was licensed in Europe in 2001, is immunogenic in young children and covers serotypes $4,6 \mathrm{~B}, 9 \mathrm{~V}$, $14,18 \mathrm{C}, 19 \mathrm{~F}$ and $23 \mathrm{~F}$. These serotypes account for between $43 \%$ and $75 \%$ of IPD in children under the age of 18 years in Western Europe [5].

Introduction of PCV in the United States in 2000 led to a dramatic decrease in those IPD that are due to vaccine serotypes, and an overall decrease of $80 \%$ in all IPD in children under the age of five years [6-8]. PCV vaccination of children had also a beneficial impact in older unvaccinated cohorts $[6,7]$. This herd effect is attributed to the reduction of pneumococcal carriage in the oropharynx of young children after PCV vaccination, reducing the transmission of vaccine-type pneumococcal strains to unvaccinated children and adults [9]. A slight increase in IPD due to non-vaccine serotypes was observed in American children after the introduction of PVC $[7,8]$. This did not significantly affect the overall reduction in pneumococcal disease incidence in American children [7,8], but has a been found to negatively affect the impact of PCV vaccination in high risk populations such as native Alaskan children [10].

In France, PCV has been recommended since 2003 for children under the age of two years who are at risk due to medical or living conditions (children in day care with at least two other children for more than four hours per week, children in families with more than two children, or children breast-fed for less than two months [11]). $79 \%$ to $89 \%$ of children under two years fall in this category in France [12]. Since June 2006, PCV vaccination has been extended to all children under the age of two years [13]. The French vaccination schedule for PCV contains three doses at the ages of two, three, and four months, administered together with the vaccines against diphtheria, tetanus, poliomyelitis, pertussis and Haemophilus influenzae type b, and then a booster at the age of 12-15 months.

The impact of PCV on IPD incidence at the national level has not been assessed in France. Moreover, as the serotype coverage of PCV appears to be lower in Europe than in North America, it is of particular interest to analyse the impact of this vaccine on pneumococcal incidence and serotype distribution in France and in other European countries.

We used surveillance data to evaluate the effect of PCV vaccine recommendations for children at risk, a definition that encompasses the majority of each birth cohort, on the incidence of pneumococcal invasive disease and serotype distribution in 2006, four years after the introduction of PCV. 


\section{Methods}

\section{Data collection}

Pneumococcal surveillance in France relies on two hospitalbased laboratory surveillance networks, Epibac and the National Reference Centre for Pneumococci (NRCP) network. Since 1987, Epibac, a national hospital-based laboratory network, has collected data on six severe invasive bacterial diseases including S. pneumoniae. Pneumococcal invasive cases are defined as the isolation of $S$. pneumoniae from blood (bacteraemia) or cerebrospinal fluid (meningitis). The participating hospitallaboratories collect information on pneumococcal invasive cases prospectively and report annually to the Institut de Veille Sanitaire. The collected data include age, sex, and site of isolation. In 2006, 307 laboratories participated, covering $79 \%$ of the French acute care hospital admissions.

Since 2001, all pneumococcal strains isolated from cerebrospinal fluid and from blood in children under 15 years-old have been collected from hospital-laboratories and sent to the NRCP by 22 regional laboratories organised into a pneumococcal surveillance regional scheme (Observatoires Régionaux des Pneumocoques). NRCP serotyped all collected strains using latex particles sensitized with a panel of antisera that was purchased from the Statens Serum Institut (Copenhagen, Denmark) and allowed to determine 90 serotypes. Pneumococcal strains with known serotypes from the Statens Serum Institut and from the NRCP collection were used as internal quality controls.

\section{Data analysis}

The annual incidence of pneumococcal bacteraemia and meningitis cases was calculated using the number of cases reported to the Epibac network as the numerator and the French population covered by Epibac participating hospitals as the denominator. The latter was estimated from the proportion of national public and private acute-care hospital admissions covered by the participating laboratories. This proportion was computed using the National Hospital Annual Activities Database which is an exhaustive source of information regarding inpatient hospital stays, managed by the Directorate for Research, Studies, Evaluation and Statistics (DREES) at the Ministry of Health. French population data is issued each year by the National Institute for Statistics and Economical Studies (INSEE).

Age-specific incidence rates were calculated in the same way using INSEE population data by age. Serotype/age-specific incidence rates for pneumococcal bacteraemia and meningitis were estimated by applying the age distribution of pneumococcal serotypes from the NRCP to age-specific incidence rates. For this analysis, serotypes 4, 6B, 9V, 14, 18C, 19F and 23F were grouped as vaccine serotypes (VT), and other serotypes as non-vaccine serotypes (NVT).

Data from 2001 and 2002, representing the pre-vaccination situation, were aggregated.

Confidence intervals $(\mathrm{Cl})$ for incidence rates were estimated using Poisson distribution. Differences in age-specific and serotype/ age-specific pneumococcal bacteraemia and meningitis incidence rates between 2001/2002 and 2006 were tested using Fisher's test for binomial data. Statistical analysis was performed using Stata 9.0 (Stata Corporation, College Station, Texas).
Due to the very recent introduction of the PCV vaccine into the immunisation schedule in France, data are not yet available from the routine infant vaccination coverage monitoring tool, which is based on the health certificates filled in for each child at the age of 24th months. Instead we used data based on PCV sales for the trend analysis. In addition, three specific interview studies, one in 2004, one in 2006 and one in 2007, were conducted in representative samples of French mothers including 1,739, 1,008 and 1,005 mothers, respectively $[12,14]$

\section{Results}

\section{Vaccine coverage}

PCV sales increased from 0,6 to 1,6 doses per child under two years between 2003 and 2006. Coverage with three doses of PCV was estimated in three specific surveys at $27 \%$ in six month-old children in 2004 [12], at 44\% in 6-12 month-old children in 2006 and at $56 \%$ in six to 12 month-old children in 2007 [14].

\section{Pneumococcal meningitis and bacteraemia in 2001-2002}

In 2001/2002, Epibac hospital-laboratories reported 7,469 cases of pneumococcal bacteraemia and 771 cases of pneumococcal meningitis. 181 (24\%) cases of pneumococcal meningitis and 493 (7\%) cases of pneumococcal bacteraemia occurred in children under two years; 194 (25\%) cases of pneumococcal meningitis and $3,806(25 \%)$ cases of pneumococcal bacteraemia occurred in adults over the age of 64 years. The reported number of cases and the estimated incidence by age-group are presented in Tables 1 and 2. The annual incidence of IPD in France was estimated at 9.4 cases per 100,000 population $(95 \% \mathrm{Cl}[9.2,9.6])$ in $2001 / 2002$.

Evolution of pneumococcal meningitis and bacteraemia incidence

\section{by age from 2001/2002 to 2006}

From 2001/2002 to 2006, pneumococcal meningitis in children under two years decreased from 8.0 to 6.0 cases per 100,000 population, a decline of $25 \%(95 \% \mathrm{Cl}[2,43], \mathrm{p}=0,04)$, and pneumococcal bacteraemia decreased from 21.8 to 17.5 cases per 100,000 population, a decline of $20 \%(95 \% \mathrm{Cl}[6,32], \mathrm{p}=0,04)$. In the same period, pneumococcal meningitis incidence showed a not statistically significant increase from 0.69 to 0.73 cases per 100,000 population $(+6 \% 95 \% \mathrm{Cl}[-7,21])$ and pneumococcal bacteraemia incidence showed a statistically significant increase from 8.2 to 9.0 cases per 100,000 population $(+11 \% 95 \% \mathrm{Cl}$ $[6,15]$ ) in older children and adults (Tables 1 and 2 ).

\section{Incidence by serotype}

In children under two years, the overall decrease in pneumococcal meningitis and bacteraemia incidences was associated with a shift in serotype distribution, NVT pneumococcal meningitis and bacteraemia cases partially replacing VT pneumococcal meningitis and bacteraemia cases (Figures 1 and 2). VT pneumococcal meningitis incidence decreased from 5.6 to 1.0 cases per 100,000 population, a $81 \%$ decline $\left(95 \% \mathrm{Cl}[67,89], \mathrm{p}<10^{-3}\right)$ and VT pneumococcal bacteraemia decreased from 14.8 to 5.3 cases per 100,000 population, a $64 \%$ decline $(95 \% \mathrm{Cl}[53,72]$, $\mathrm{p}<10^{-3}$ ) from $2001 / 2002$ to 2006 . In the same time period, NVT pneumococcal meningitis incidence increased from 2.4 to 4.9 cases per 100,000 population, a $102 \%$ rise $(95 \% \mathrm{Cl}[41,191]$ $\mathrm{p}<10^{-3}$ ) and pneumococcal bacteraemia increased from 7.0 to 12.2 cases per 100,000 population, a $74 \%$ rise $(95 \% \mathrm{Cl}[39,114]$, $\left.\mathrm{p}<10^{-3}\right)$ 


\section{Evolution of serotype distribution}

We determined the serotype for 156 pneumococcal strains isolated from meningitis cases and 246 pneumococcal strains isolated from bacteraemia cases in children under two years, in $2001 / 2002$, as well as 67 strains isolated from meningitis and 99 isolated from bacteraemia in 2006.

In children under the age of two years, VT pneumococcal strains accounted for $68 \%$ (274/402) of the serotyped strains isolated from pneumococcal meningitis and bacteraemia cases in 2001/2002 and $25 \%(42 / 166)$ in 2006.

Among NVT pneumococcal meningitis and bacteraemia cases that occurred in children under two years in 2006, serotypes 19A and $7 \mathrm{~F}$ were the most frequent (Figure 3 ). Together they accounted for $37 \%$ of pneumococcal meningitis and bacteraemia. From $2001 / 2002$ to 2006 , the proportion of 19 A strains increased from $8 \%$ to $19 \%$ in meningitis cases $(p=0,03)$ and from $11 \%$ to

T A B L E 1

Reported pneumococcal meningitis cases and estimated pneumococcal meningitis incidence by age in 2001/2002 and 2006, France (source: Epibac)

\begin{tabular}{|c|c|c|c|c|c|c|c|}
\hline \multirow{3}{*}{$\begin{array}{l}\text { Age group } \\
<2 \text { years }\end{array}$} & \multicolumn{2}{|c|}{ No. of reported cases } & \multicolumn{2}{|c|}{ Cases/100,000/year } & \multirow{2}{*}{\multicolumn{2}{|c|}{$\frac{\text { Incidence rate ratio, CI 95\% }}{2006 \text { vs. } 2001 / 2002}$}} & \multirow{3}{*}{$\frac{p}{0.036}$} \\
\hline & $2001 / 2002$ & 2006 & $2001 / 2002$ & 2006 & & & \\
\hline & 181 & 74 & 8.0 & 6.0 & 0.75 & {$[0.57,0.98]$} & \\
\hline $2-15$ years & 74 & 41 & 0.5 & 0.5 & 1.02 & {$[0.70,1.50]$} & 0.922 \\
\hline $16-64$ years & 322 & 199 & 0.6 & 0.6 & 1.11 & {$[0.93,1.32]$} & 0.254 \\
\hline$>64$ years & 194 & 106 & 1.4 & 1.3 & 0.97 & {$[0.77,1.23]$} & 0.857 \\
\hline Total & 771 & 420 & 0.9 & 0.9 & 0.98 & {$[0.87,1.11]$} & 0.785 \\
\hline
\end{tabular}

\section{T A B L E 2}

Reported pneumococcal bacteraemia cases and estimated pneumococcal bacteraemia incidence by age in 2001/2002 and 2006, France (source: Epibac)

\begin{tabular}{|c|c|c|c|c|c|c|c|}
\hline \multirow{3}{*}{$\begin{array}{l}\text { Age group } \\
<2 \text { years }\end{array}$} & \multicolumn{2}{|c|}{ No. of reported cases } & \multicolumn{2}{|c|}{ Cases/100,000/year } & \multirow{2}{*}{\multicolumn{2}{|c|}{$\begin{array}{c}\text { Incidence rate ratio, CI 95\% } \\
2006 \text { vs. 2001/2002 }\end{array}$}} & \multirow{3}{*}{$\frac{p}{0.007}$} \\
\hline & \multirow{2}{*}{$\frac{2001 / 2002}{493}$} & \multirow{2}{*}{$\frac{2006}{217}$} & \multirow{2}{*}{$\frac{2001 / 2002}{21.8}$} & \multirow{2}{*}{$\begin{array}{r}2006 \\
17.5\end{array}$} & & & \\
\hline & & & & & 0.80 & {$[0.68,0.94]$} & \\
\hline $2-15$ years & 416 & 274 & 2.7 & 3.3 & 1.22 & {$[1.04,1.42]$} & 0.013 \\
\hline 16 - 64 years & 2,754 & 1,681 & $4 ., 9$ & 5.4 & 1.10 & {$[1.03,1.16]$} & 0.003 \\
\hline$>64$ years & 3,806 & 2,329 & 26.8 & 29.0 & 1.08 & {$[1.03,1.14]$} & 0.003 \\
\hline Total & 7,469 & 4,501 & 8.5 & 9.2 & 1.08 & {$[1.05,1.13]$} & 0.000 \\
\hline
\end{tabular}

\section{F I G U R E 1}

Estimated pneumococcal meningitis incidence by serotype in children under two years of age, France 2001-2006

(source: Epibac-NRCP)

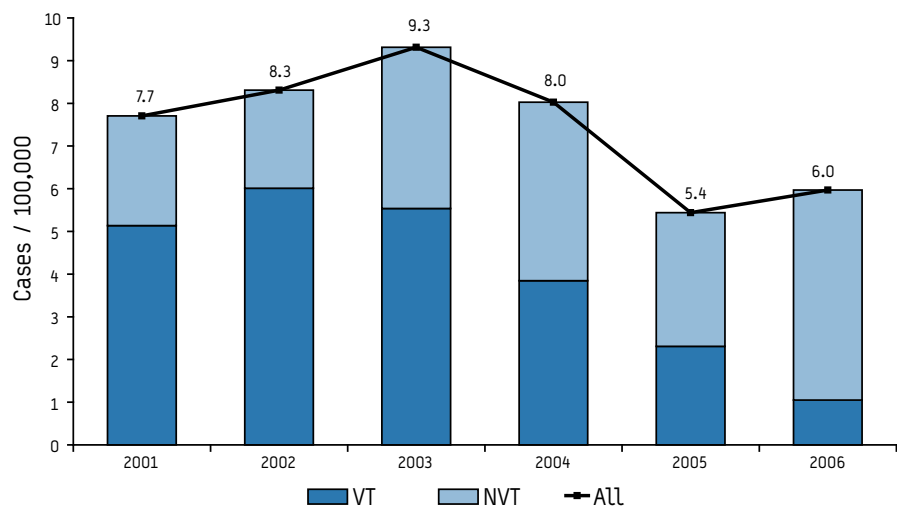

VT: vaccine serotypes; NVT: non-vaccine serotypes

\section{F I G U R E}

Estimated pneumococcal bacteraemia incidence by serotype in children under two years of age, France 2001-2006

(source: Epibac-NRCP)

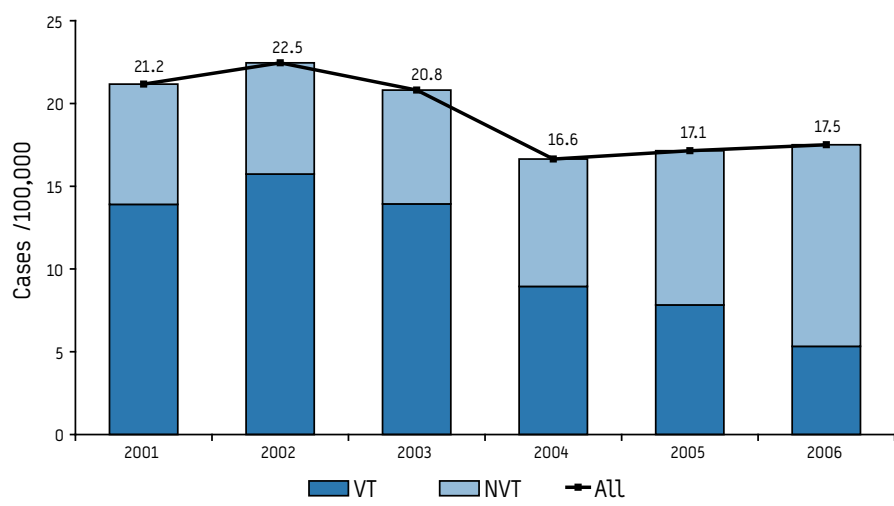

VT: vaccine serotypes; NVT: non-vaccine serotypes 
$27 \%$ in bacteraemia cases $\left(p<10^{-3}\right)$; the proportion of $7 \mathrm{~F}$ strains increased from $3 \%$ to $18 \%$ in meningitis cases $\left(p<10^{-3}\right)$ and from $1 \%$ to $10 \%$ in bacteraemia cases $\left(p<10^{-3}\right)$. Other non-vaccine serotypes accounted for less than $8 \%$ of pneumococcal meningitis and bacteraemia cases in children under two years in 2006.

From $2001 / 2002$ to 2006 , the incidence of pneumococcal meningitis and bacteraemia caused by each of the seven vaccine serotypes decreased. The incidence of pneumococcal meningitis and bacteraemia due to serotypes which are not included in the vaccine but are part of the same serogroup as a vaccine serotype with the exception of serotype 19A, i.e. serotypes 23B, 6A, 18B, $9 \mathrm{~N}$, and $23 \mathrm{~A}$ - remained unchanged (Figure 3 ).

\section{Discussion}

French recommendations for PCV vaccination in 2003 included a large proportion of French children under the age of two years, while other European countries targeted only high risk children [4]. Between 2005 and 2006, vaccination with PCV has been extended to all children under two years in France as well as in other European countries such as Belgium, England, Germany, Luxembourg, the Netherlands, and Norway [15]. The early recommendations and the fact that the French surveillance for invasive pneumococcal diseases allows the analysis of trends in incidence and serotype distribution provided an opportunity to analyse the impact of PCV introduction in France. It is the second analysis of this kind in a European country at the national level following the analysis published this year from Norway [16].
Although PCV introduction in France was associated with a 71\% decrease in vaccine-type IPD incidence between 2001/2002 and 2006 in children under two years, the overall decrease of IPD in this age group was only $21 \%(95 \% \mathrm{Cl}[10,31])$. The fact that the decline was observed only in children under two years and only for cases due to vaccine serotype strains strongly argues for a role of $\mathrm{PCV}$ vaccination in this evolution.

\section{Impact of PCV vaccination on IPD incidence}

The $21 \%$ decline of the disease in French children is far below the $77 \%$ reduction observed in children under the age of five years in the regions covered by the United States (US) Centers for Disease Control and Prevention's 'Active Bacterial Core surveillance' in 2005 and the $52 \%$ reduction observed in children under two years in Norway between 2004/2005 and 2007 [8,16]. Moreover, an indirect benefit of PCV vaccination in other age-groups has so far not been observed in France. The limited estimated vaccination coverage for PCV, below $60 \%$ in 2006 , could explain in part this modest impact of PCV vaccination. Although PCV vaccine coverage has improved in the recent years, it remains well below the usual vaccine coverage for infants in France [14]. Expansion of the PCV vaccination coverage should lead to a further reduction in the IPD that are caused by vaccine serotypes in children and, through indirect effects, also in adults.

The overall reduction in IPD decrease that we found in young children is in agreement with the results reported by a French network of paediatricians which indicate a $28 \%$ decrease in the number of pneumococcal meningitides in 2-24 month-old children

\section{F I G U R E 3}

Estimated incidence of pneumococcal meningitis and bacteraemia by serotype in children under the age of two years, evolution from 2001/2002 to 2006, France (source: Epibac-NRCP)

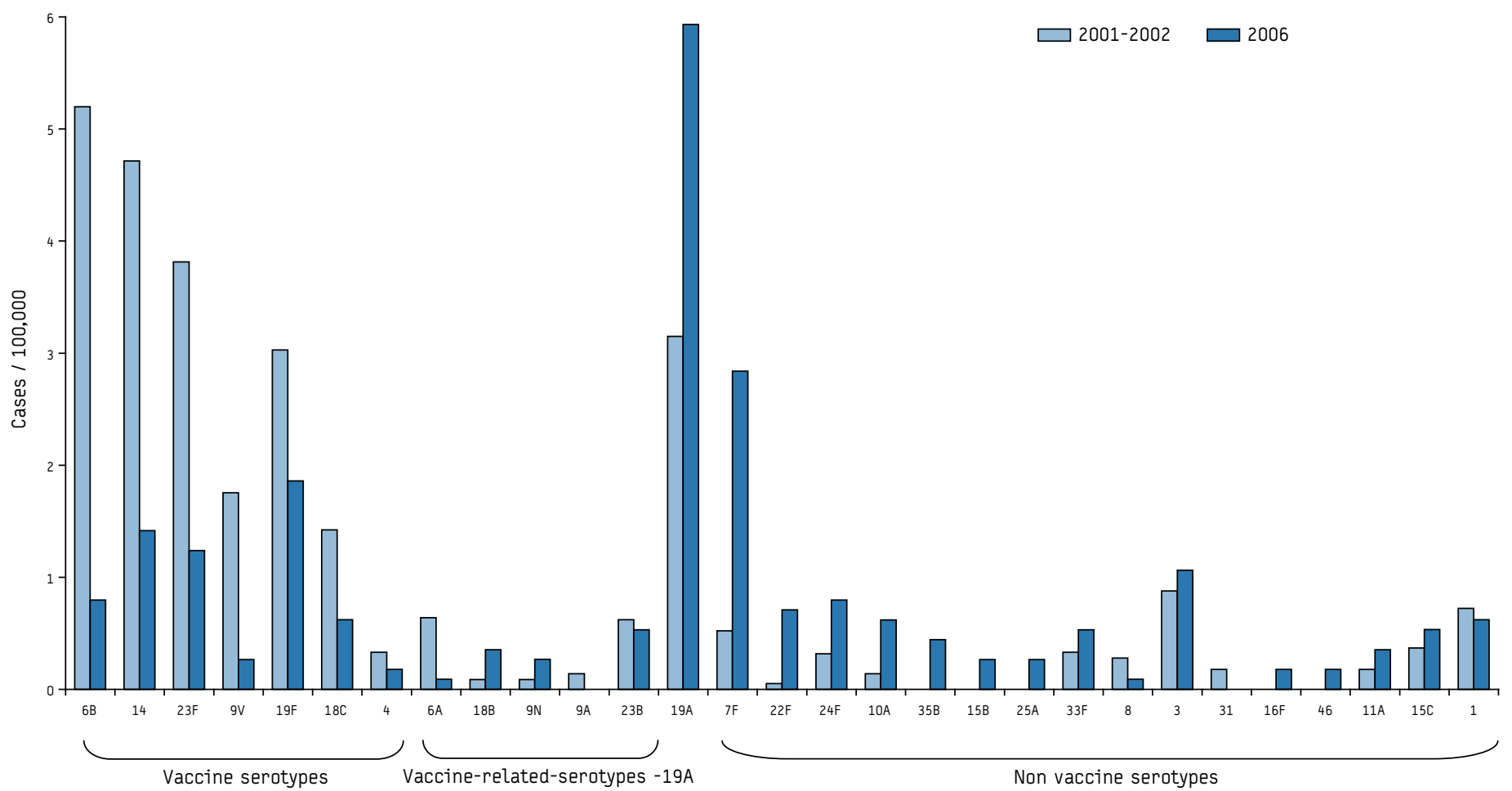


between 2001/2002 and 2005 [17]. Another survey conducted in 18 hospitals in northern France found a much greater reduction $(82 \%)$ in pneumococcal meningitis incidence in children under two years between 2002 and 2005 than was found in the above and in our results [18]. A high PCV coverage in the northern region of France and the small number of cases involved in this retrospective survey $(n<8$ in 2005) are possible explanations for these differences.

\section{Serotype replacement}

The $71 \%$ reduction in IPD incidence due to VT strains was associated with a $85 \%$ rise in cases due to NVT strains in children under two years from 2001/2002 to 2006 . The magnitude of this replacement impacted the overall effect of PCV vaccination: IPD incidence in French young children decreased between 2001/2002 and 2005 but did not decrease further from 2005 to 2006 despite a $20 \%$ rise in PCV sales. During that later period, the decrease in cases due to VT strains was balanced by an increase of the same magnitude in cases due to NVT strains. Replacement of VT by NVT strains has been observed to a smaller extent also in American children following PCV introduction. The increase of NVT pneumococcal disease in children under five years in the US estimated from 'Active Bacterial Core surveillance' data between $1998 / 1999$ and 2005 was only $29 \%$ [8]. That this increase was higher in France may be due to the lower PCV serotype coverage in young children in France compared to the US. Before PCV introduction, 68\% of IPD in children under two years were caused by PCV serotypes in 2001/2002 in France, compared to $83 \%$ in children under five years in 1998/1999 in the US [7].

Among NVT strains, two single serotypes - 19A and 7F accounted for $37 \%$ of pneumococcal strains in 2006 in France. In the US, the 19A serotype has been found to be the predominant serotype in pneumococcal invasive cases in the years following PCV implementation, accounting for $40 \%$ of cases in children under the age of five 5 years in 2005, according to the results of the 'Active Bacterial Core surveillance' [8].

No decline in IPD was observed in older children and adults; on the contrary we identified a small but significant increase. However, as this trend had already been observed from 1998 to 2002 before the introduction of PCV in France, the possible contribution of vaccination to the increase cannot be conclusively assessed [19].

The evolution of pneumococcal invasive incidence in children in France can be compared with the situation observed in different areas of Spain after PCV introduction. Four regional Spanish studies were performed with the following results: no change in IPD incidence in a Barcelona district between 1999/2001 and 2002/2004 [20], a decrease in the Basque region between 2000/2001 and 2004/2005 [21] and in the Basque and Navarre regions between 1998/2001 and 2003 [22], and even an increase in pneumococcal invasive cases in Barcelona between 1997/2001 and 2002/2006 [23]. The reasons given by the investigators for this limited impact of PCV on IPD incidence refer to the conditions of PCV introduction in Spain: Vaccine coverage was low in Spain, where PCV is not subsidised, and the serotype coverage of PCV was significantly lower than the PCV coverage in the US (43\% in the Navarre region) [20,22-24]. An increase in the frequency of pneumococcal invasive cases due to non-vaccine strains after PCV introduction was also found in three of theses studies $[20,23,24]$.

\section{Strengths and limitations of the study}

We are confident about our incidence estimates because of the high and sustained coverage of the Epibac laboratory network combined with extrapolations made on a reliable source of information (the French national Hospital Annual Statistic database). Furthermore, we regularly monitored the reporting of cases by the participating laboratories through three sources capture-recapture analysis to ensure the exhaustiveness of the reports. The rate of underreporting were estimated at between $10 \%$ and $20 \%$ in these analyses $[25,26]$.

We cannot completely exclude a change in the rate of pneumococcal case reporting in the last years. However, significant changes in reporting for pneumococcal cases alone are unlikely due to the following reasons: Firstly, the reporting rate of other bacterial diseases included in Epibac surveillance has not changed until 2005 as shown by a recent three sources capture-recapture analysis for invasive meningococcal diseases [26]; secondly, pneumococcal data show opposite trends for VT- and NVT-related cases; and thirdly, the cases are reported, by the vast majority of participating laboratories, through automatic extraction of microbiology isolates registration.

Incidence and serotype data are issued from two networks whose regional coverage is not identical. This may have introduced biases in the estimation of serotype/age-specific incidence evolution. However, each both networks covers more than 300 hospitals localised in all French regions, and the PCV serotype coverage did not vary with the geographical origin of pneumococcal strains (data not shown).

The evolution of individual serotypes should be interpreted with caution given the small number of strains involved in the 2006 analysis. Emergence of serotypes $19 \mathrm{~A}$ and $7 \mathrm{~F}$ may not be due to PCV introduction alone, as changes in serotypes distribution can also occur for other reasons than vaccination pressure. The findings of this early analysis must be seen as a preliminary description of the PCV impact in France. IPD evolution and the extent of serotype replacement will be closely monitored in the next years through ongoing epidemiological and bacteriological surveillance.

\section{Conclusion}

In conclusion, PCV introduction was followed by a significant decrease in IPD in young children in France. Further improvement of PCV coverage should further increase the positive impact of PCV on vaccine-type pneumococcal invasive diseases in both children and adults in the next years, if a positive herd immunity effect is observed. If, on the other hand, the partial substitution of the cases that are caused by vaccine serotypes with cases caused by non-vaccine serotypes, that was observed in our early analysis in young children, is confirmed in the coming years, this would lead to a reduction of the positive impact of PCV vaccination in France.

Theses results emphasise the need for ongoing surveillance of the pneumococcal disease incidence and serotype in countries introducing PCV. The imminent availability of pneumococcal vaccines covering a broader range of the serotypes implicated in IPD in young children could limit the effect of serotype replacement and improve the impact of immunisation on IPD. 


\section{Acknowledgements:}

We gratefully acknowledge Ms Edith Laurent (InVS), the microbiologists of the Epibac network (http://www.invs.sante.fr/surveillance/index.htm) and the members of the Observatoires régionaux des pneumocoques network (http://www.invs.sante.fr/beh/2006/01/participants_22_orp.pdf) for collecting and providing the data on pneumococcal invasive disease since 2001, and Dr Jean Claude Desenclos for his advice and review of this manuscript.

\section{References}

1. Georges S, Perrocheau A, Laurent E, Lévy-Bruhl D. Infections invasives à $H$. influenzae, L. monocytogenes, N. meningitidis, S. pneumoniae, S. agalactia et S. pyogenes en France en 2001-2002. Bull Epidemiol Hebd. 2004;34:165-8. Available from: http://www.invs.sante.fr/beh/2004/34/index.htm

2. Perrocheau A, De Benoist AC, Six C, Goulet V, Decludt B, Levy-Bruhl D. Epidemiology of bacterial meningitis in France in 1999. Ann Med Interne (Paris). 2002;153:311-7.

3. Bingen E, Levy C, de la Rocque F, Boucherat M, Varon E, Alonso JM, et al. Bacterial meningitis in children: a French prospective study. Clin Infect Dis. 2005;41(7):1059-63.

4. Pebody R, Leino T, Nohynek H, Hellenbrand W, Salmaso S, Ruutu P. Pneumococcal vaccination policy in Europe. Euro Surveill. 2005;10(9):pii=564. Available from: http://www.eurosurveillance.org/ViewArticle.aspx?ArticleId $=564$

5. Jefferson T, Ferroni E, Curtale F, Giorgi Rossi P, Borgia P. Streptococcus pneumoniae in western Europe: serotype distribution and incidence in children less than 2 years old. The Lancet Infect Dis. 2006;6(7):405-10.

6. Centers for Disease Control and Prevention (CDC). Direct and indirect effects of routine vaccination of children with 7-valent pneumococcal conjugate vaccine on incidence of invasive pneumococcal disease--United States, 1998-2003. MMWR Morb Mortal Wkly Rep. 2005;54(36):893-7.

7. Hicks LA, Harrison LH, Flannery B, Hadler JL, Schaffner W, Craig AS, et al. Incidence of pneumococcal disease due to non-pneumococcal conjugate vaccine (PCV7) serotypes in the United States during the era of widespread PCV7 vaccination, 1998-2004. J Infect Dis. 2007;196(9):1346-54.

8. Centers for Disease Control and Prevention (CDC). Invasive Pneumococcal Disease in Children 5 Years After Conjugate Vaccine Introduction -Eight States, 1998-2005. MMWR Morb Mortal Wkly Rep. 2008;57(6):144-8.

9. O'Brien KL, Millar EV, Zell ER, Bronsdon M, Weatherholtz R, Reid R, et al. Effect of pneumococcal conjugate vaccine on nasopharyngeal colonization among immunized and unimmunized children in a community-randomized trial. Infect Dis. 2007;196(8):1211-20.

10. Singleton RJ, Hennessy TW, Bulkow LR, Hammitt LL, Zulz T, Hurlburt DA, et al. Invasive pneumococcal disease caused by nonvaccine serotypes among alaska native children with high levels of 7 -valent pneumococcal conjugate vaccine coverage. JAMA. 2007;297(16):1784-92.

11. Conseil supérieur d'hygiène publique de France, section des maladies transmissibles. Avis du 8 mars 2002 relatif à la vaccination par le vaccin pneumococcique heptavalent conjugué - Prevenar. Available from: http://www. sante.gouv.fr/htm/dossiers/cshpf/cs231.htm

12. Cohen R, Gaudelus J, Pexoito 0 . Vaccin antipneumococcique conjugué: estimation de la population cible. Enquête auprès de 1739 mères. Médecine \& enfance. 2005;25(4):237-42.

13. Conseil supérieur d'hygiène publique de France, section des maladies transmissibles. Avis du 19 mai 2006 relatif à la vaccination par le vaccin anti-pneumococcique conjugué chez les enfants de moins de deux ans et les enfants de deux à cinq ans. Available from : http://www.invs.sante.fr/ beh/2006/29 $30 /$ beh 2930 2006.pdf

14. Gaudelus J, Cohen R, Hovart J. Couverture vaccinale du vaccin pneumococcique heptavalent conjugué en 2007, comparaison avec les années précédentes et les autres vaccins pédiatriques: analyse des carnets de santé. Médecine et Enfance, 2007;27(5):1-4. Available from: http://www.medecine-et-enfance. net/

15. Lopalco PL, Editorial team. Use of 7-valent pneumococcal conjugate vaccine in EU. Euro Surveill. 2006;11(49):pii=3092. Available from: http://www. eurosurveillance.org/ViewArticle. aspx?ArticleId $=3092$

16. Vestrheim DF, Løvoll O, Aaberge IS, Caugant DA, Høiby EA, Bakke H, et al. Effectiveness of a $2+1$ dose schedule pneumococcal conjugate vaccination programme on invasive pneumococcal disease among children in Norway. Vaccine. 2008;26(26):3277-3281.

17. Bingen E, Levy C, Varon E, de La Rocque F, Boucherat M, d'Athis P, et al. Pneumococcal meningitis in the era of pneumococcal conjugate vaccine implementation. Eur J Clin Microbiol Infect Dis. 2008 Mar;27(3):191-9.
18. Dubos F, Marechal I, Husson M O, Courouble, Aurel M, Martinot A, et al. Decline in pneumococcal meningitis after the introduction of the heptavalentpneumococcal conjugate vaccine in northern France. Arch Dis Child. 2007; 92(11): 1009-12.

19. Lepoutre A., Georges S., Varon E., Lévy-Bruhl D, et les microbiologistes du réseau Epibac. Evolution des infections invasives à pneumocoque, France 2005 Bull Epidemiol Hebd. 2007;5:37-9. Available from: http://www.invs.sante.fr/ beh/2007/05/index.htm

20. Calbo E, Díaz A, Cañadell E, Fábrega J, Uriz S, Xercavins M, et al. Invasive pneumococcal disease among children in a health district of Barcelona: early impact of pneumococcal conjugate vaccine. Clin Microbiol Infect. 2006;12(9):867-72.

21. Benito-Fernandez J, Raso SM, Pocheville-Gurutzeta I, SanchezEtxaniz J, Azcunaga-Santibanez B, Capape-Zache S. Pneumococcal bacteremia among infants with fever without known source before and after introduction of pneumococcal conjugate vaccine in the Basque Country of Spain. Pediatr Infect Dis J. 2007;26(8):667-71.

22. Aristegui J, Bernaola E, Pocheville I, García C, Arranz L, Durán G, et al. Reduction in pediatric invasive pneumococcal disease in the Basque Country and Navarre, Spain, after introduction of the heptavalent pneumococcal conjugate vaccine. Eur J Clin Microbiol Infect Dis. 2007;26(5):303-10.

23. Munoz-Almagro C, Jordan I, Gene A, Latorre C, Garcia-Garcia JJ, Pallares R. Emergence of invasive pneumococcal disease caused by nonvaccine serotypes in the era of 7-valent conjugate vaccine. Clin Infect Dis. 2008;46(2):174-82.

24. Barricarte A, Castilla J, Gil-Setas A, Torroba L, Navarro-Alonso JA, Irisarr $F$, et al. Effectiveness of the 7-valent pneumococcal conjugate vaccine: population-based case-control study. Clin Infect Dis. 2007;44(11):1436-41.

25. Perrocheau A, Doyle A, Bernillon P, Varon E, le groupe des Observatoires Régionaux du Pneumocoque, De la Rocque F, et al. Estimation du nombre total de méningites à pneumocoque de l’enfant par la méthode capture- recapture à 3 sources, France, 2001-2002. Bull Epidemiol Hebd. 2006:2-3,16-9. Available from; http://www.invs.sante.fr/beh/2006/02_03/index.htm

26. Berger F, Bernillon P, Parent du Chatelet I, Taha M-K, Georges S, Perrocheau $A$, et al. Three-sources capture-recapture analysis to evaluate the comprehensiveness of reporting invasive meningococcal infections in France, 2005. [Poster communication] 9th Meeting of the European Monitoring Group on Menongococci, 30 May-1 June 2007, Rome, Italy.

This article was published on 28 August 2008

Citation style for this article: Lepoutre A, Varon E, Georges S, Gutmann L, Lévy-Bruhl D. Impact of infant pneumococcal vaccination on invasive pneumococcal diseases in France, 2001-2006. Euro Surveill. 2008;13(35):pii=18962. Available online: http://www. eurosurveillance.org/ViewArticle.aspx?ArticleId $=18962$ 